\title{
RECONSTRUCTION OF THE ORBITAL SKELETON USING COMPUTER- AIDED INDIVIDUALIZED TITANIUM MESH COMPARED TO CONVENTIONAL TITANIUM MESH
}

\author{
Omaima M. Saqr ${ }^{1 *} M S c$, Ahmed R. Kotb ${ }^{2} P h D$, Ahmed S. EL-Mahallawy² PhD, Riham M. El \\ Dibany $^{2} P h D$, Mahmoud H. Moursy ${ }^{3} P h D$
}

\begin{abstract} function of infraorbital nerve

* Corresponding author:

E-mail:drsakr2009@hotmail.com,
\end{abstract}

INTRODUCTION: Orbital reconstruction is one of the most challenging tasks for the surgeon who treats craniofacial trauma. Suboptimal outcomes may lead to debilitating morbidity with significant emotional, functional, and occupational deficits

OBJECTIVES: This study was designed to compare the efficacy of the computer-aided fabricated individual titanium mesh and conventional titanium mesh in orbital skeleton reconstruction in cases of infraorbital dysesthesia.

MATERIALS AND METHODS: The study population included 20 patients with orbital skeleton fractures. The sample was selected conveniently to fulfill a list of inclusion criteria. The selected participants were randomly allocated into two equal groups, each group included 10 patients. In the course of a surgical treatment, individually designed titanium mesh were manufactured by application of computer- aided design in combination with rapid prototyping technologies (CAD/RP). Preoperative analysis and postoperative monitoring were conducted to evaluate their success based on restoration of infraorbital nerve function that was evaluated by pin prick test and electrical pulp testing. RESULTS: Regarding postoperative clinical evaluation, group 1(study group) was superior to that of group 2 (control group) in restoring the

CONCLUSIONS: computer aided titanium mesh was an accurate option in reconstructions of traumatic orbital wall fractures.

KEYWORDS: Computer aided titanium mesh, Prefabricated titanium mesh, 3D printing.

1- MD Oral and Maxillofacial Surgery, Faculty of Dentistry, Alexandria University, Egypt.

2- Professor of Oral and Maxillofacial Surgery, Faculty of Dentistry, Alexandria University, Egypt.

3- Professor of Ophthalmology Faculty of Medicine, Alexandria University, Egypt.

\section{INTRODUCTION}

Orbital reconstruction is one of the most challenging tasks for the surgeon who treats craniofacial trauma. Suboptimal outcomes may lead to debilitating morbidity with significant emotional, functional, and occupational deficits. These deficits can include diminished visual acuity, diplopia, and loss of depth perception, chronic or severe pain, as well as parasthesia of the infraorbital nerve. Repair of orbital fractures is challenging because of the complexity and variability of the anatomy (1).

Computers are being used increasingly as a supportive tool for diagnosis and surgical treatment planning in craniomaxillofacial field (2). They are used in conjunction with digital imaging techniques such as computed tomography to improve visualization of anatomical and physiologic conditions (3). Computer-aided design has gained popularity in clinical practice, and the advent of rapid prototyping technology has further enhanced the quality and predictability of surgical outcomes. Surgeons can efficiently and precisely target fracture restorations, based on three-dimensional models generated from a computed tomographic scan. Precise preoperative planning simulation on a computer is possible (4).

Rapid prototyping is a class of technologies that can construct physical models from computer-aided design via three dimensional printers. Although rapid prototyping has been used in the field of industrial design for over three decades, only in the last decade have applications been developed for medicine. There are numerous rapid prototyping technologies; the most commonly used for medical applications is stereolithography. It is a process by which liquid polymer media contained in a vat is "cured" via laser into solid resin, which comprises both the three dimensional model and support pillars (which help maintain the structural integrity of the model) is being created. These models are accurate to 0.1 $\mathrm{mm}$, and the build time is dependent on the complexity of the structure being printed (5).

Therefore, the current study was designed to highlight the precision and accuracy obtained with patient specific titanium mesh for orbital reconstruction designed on the basis of volumetric analysis of orbital computed tomographic scans (CT) using virtual planning, computerized designing and manufacturing and stereolithographic models to restore orbital volume and correct the late post-traumatic orbital deformities such as diplopia, enophthalmos and infra orbital parenthesis.

\section{MATERIALS AND METHODS \\ Study design}

This study was carried out as a controlled clinical trial.

Study setting

This clinical trial was conducted on 20 patients of both sexes. Patients were diagnosed with unilateral orbital skeleton fracture, and they were selected from the 
Emergency room of the Oral and Maxillofacial Surgery Department, Faculty of Dentistry, Alexandria University.

Patients were instructed about the procedure that was performed and an informed consent was signed by each participant. The sample was selected conveniently to fulfill the required inclusion and exclusion criteria. The selected 20 participants were allocated randomly in to two equal groups each consisting of 10 patients.

Group 1: (study group) 10 patients underwent orbital reconstruction using the computer-aided fabricated individual titanium mesh

Group 2: (control group) 10 patient underwent orbital reconstruction using conventional titanium mesh.

\section{Criteria of patient selection}

\section{Inclusion criteria}

Patients of both sexes were included an age range from 20to 60 years suffering from unilateral orbital fractures either isolated or combined with other facial fractures combined with infraorbital nerve anesthesia or parasthesia that required reconstruction.

\section{Exclusion Criteria}

Patients excluded from the study if they present with intraocular or global hematoma, bilateral orbital fractures, and patients suffering from chronic systemic diseases such as uncontrolled diabetes, rheumatoid arthritis, metabolic bone disease, immunocompromised status.

\section{MATERIALS}

\section{Rapid prototyping orbital model material}

The model was made from Polyacrylic acid (PAA). It was cross-linked with an allyl ether of pentaerythritol and allyl ether of propylene. In a water solution at neutral $\mathrm{pH}$, PAA is an anionic polymer.

\section{Stereolithography machine}

Stereolithography (SLA) machine builds the stereo-models from liquid photosensitive polymers that solidify when exposed to ultraviolet light beam. The model was built upon a platform situated just below the surface in a vat of liquid epoxy or acrylic resin. The model was placed inside an ultraviolet oven for the final curing (6).

\section{Titanium mesh and screws}

A 120x75x0.6mm titanium mesh system was alloyed with about $6 \%$ aluminum and $4 \%$ vanadium with $1.6 \times 7 \mathrm{~mm}$ titanium screws (Medicatechnology, Cairo, Egypt). Such alloys have very high tensile strength and toughness (even at extreme temperatures). They are light in weight, have extraordinary corrosion resistance and the ability to withstand extreme temperatures.

\section{METHODS}

\section{I.Pre-operative phase}

\section{A. Pre-operative assessment}

After admission to the Oral and Maxillofacial Surgery Department all patients were subjected to the following:

\section{History}

Comprehensive medical and dental history was collected from all patients including name, age, gender, occupation, address, date, time of injury, and immediate measures.

\section{General examination}

The general condition was assessed for all patients and the vital signs were recorded (temperature, blood pressure, respiratory rate and puls).

\section{Clinical examination}

\section{a. Inspection and palpation}

All patients were subjected to full clinical examination by inspection and palpation of the fracture sites to detect the presence of edema, ecchymosis, soft tissue laceration, areas of tenderness, step deformities, mobility of the fractured segments, and ocular mobility.

\section{b. Neurosensory evaluation}

Evaluation of the sensory disturbance of infraorbital nerve was performed by the following tests:

\section{Pin prick test (7)}

- Tip of a $0.2 \mathrm{~mm}$ diameter blunted acupuncture needle was pushed against the patient's skin until the needle slightly bends (the skin was dimpled but not penetrated). The specific sites were included: mid-way of the dimensions of lower eye lid, middle of the lateral part of the nose, middle portion of the upper lip and middle of zygoma. (Fig. 1)

- The patients graded sensations were recorded in $100 \mathrm{~mm}$ visual analogue scale. Results were recorded as the difference in the VAS values between the control and injured sides.

Pulp testing of the involved ipsilateral maxillary teeth (8)

- It worked on the premise that electrical stimuli cause an ionic change across the neural membrane, thereby inducing an action potential with a rapid hopping action at the nodes of Ranvier in myelinated nerves.

- The "circuit" is completed via the patient wearing a lip clip or by touching the probe handle with his/her hand; alternatively, the operator can have one "gloveless" hand that touches the patient's skin.

- A "tingling" sensation was felt by the patient once the increasing voltage reached the pain threshold.

- The patients graded sensations were recorded in $100 \mathrm{~mm}$ visual analogue scale. Results were recorded as the difference in the VAS values between the control and injured sides. (Fig. 2)

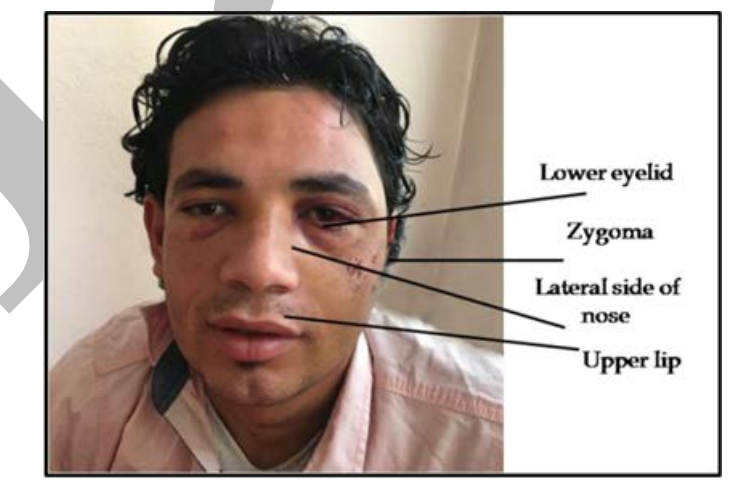

Figure (1): Pain prick test landmark

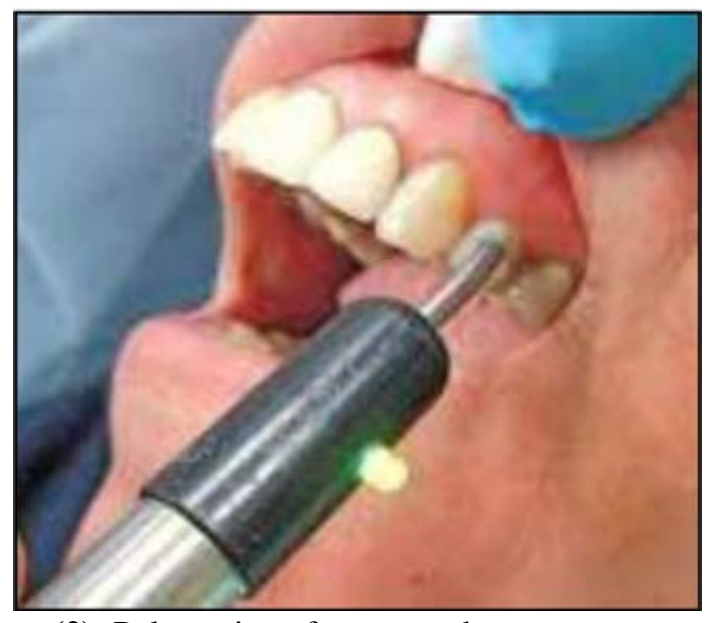

Figure (2): Pulp testing of upper teeth. 


\section{Radiographic examination}

Radiographic examination was accomplished for all patients at the time of presentation. $1 \mathrm{~mm}$ cuts, axial, coronal, sagittal and 3D reconstruction computerized tomography was taken for all patients. Axial images assess fracture of the lateral orbital wall, coronal and sagittal image assess fracture of the orbital floor, and 3D CT provides improved depiction of complex orbital fractures. They generate more spatial information and show fracture extent and fragment displacement in severe trauma with multiple fracture fragments.

2. Laboratory investigations

- Complete blood count (CBC).

- Coagulation profile: Prothrombin time (PT), partial thromboplastin time (PTT) and international normalized ratio (INR).

- Liver functions: Alanine Transaminase (ALT) and Aspartate Transaminase (AST).

- Kidney functions: Creatinine blood level, urea.

- ECG: was ordered for patients above 40 years and risk patients below 40 years.

\section{Photographs}

Photographs were taken preoperatively and post operatively to show the difference in appearance of each patient before and after surgery.

\section{B. Orbital model fabrication}

The CT imaging was performed on a 64-slice multi detector CT scanner. Volumetric data was acquired (1 mm slice thickness) and calculated by the digital imaging and communications in medicine (DICOM) format. The virtual 3D representation was created by subsequent image segmentation and using a "mirror image" of the contra lateral side of the orbit as a reference through the MIMICS software program (Materialise's Interactive Medical Image Control System, Materialise), then the data was converted to steriolithography (STL) format which is supported by various software ready for rapid prototyping and orbital model was then printed and sterilized. (Fig. 3)

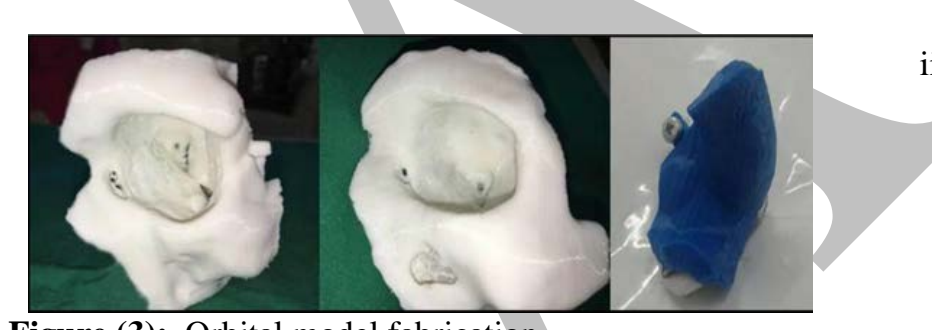

Figure (3): Orbital model fabrication

\section{Construction of the titanium mesh}

From stereolithographically produced 3D models, the orbital defect was easily seen and assessed. The orbital defect was then filled using wax to reproduce a like contour to the contralateral side and an impression was taken of both orbital cavities using silicon putty impression material. Then the orbital injury side was reproduced by pouring a hard plaster/stone model. The defect had been filled and therefore appears in its proposed reconstructed form. Using pressure flasks usually used in denture construction a layer of $0.6 \mathrm{~mm}$ medical grade titanium is then swaged onto the model of the orbital defect producing an exact replica of the proposed orbital contour. The titanium sheet may then be trimmed to allow sufficient overlap and the positioning of a flange for screw fixation.

\section{Pre-operative medications}

Prophylactic antibiotic therapy in the form of Clindamycin 600mg (Dalacin c phosphate, Sigma, Egypt.) every 12 hours was administered pre-operatively commencing upon admission in addition to anti-inflammatory drug, Ibuprofen 400mg every 8 hours (Brufen 400, Abbott. Egypt.).

\section{II.Operative phase}

A. Anesthesia

- All operations were performed under general anesthesia and strict aseptic conditions in the theater.

- Nasotracheal, orotracheal or submental intubation were performed according to the association with other facial fractures.

B- Surgical procedures

- The patient was placed in a supine position.

- Intraoperative medications including Amoxicillin /Clavulanic acid 1.2GM (Augmentin 1,2 gm vial: $1000 \mathrm{mg}$ Amoxicillin and $200 \mathrm{mg}$ Clavulanic acid. Galaxo Simith Kline, Egypt.) intravenous injections, dexamethasone phosphate $8 \mathrm{mg}$ (Epidron ampule; $8 \mathrm{mg} / 12 \mathrm{ml}$. E.L.P.L.C.O, Egypt.) intravenously, were given to the patient.

- Patients were scrubbed and draped according to oral and maxillofacial surgery protocol.

- The cornea was protected by application of Polymyxin/tetracycline eye ointment (Teramycin100IU/g, Polymyxin and $0.5 \%$ oxytetracycline. pfeizer, Egypt) in both eyes and a temporary tarsorrhaphy.

- Vasoconstrictors (Adrenaline 1 ampule 1: $200000 \mathrm{IU} / \mathrm{ml}$, Scied. Egypt) were used according to the location of the various incision lines for hemostasis.

- The approach to the fracture site depended upon the type of injury;

i.The existing laceration was used to access directly the area of fractured area or extend the laceration to attain enough access to the fractured area, placing additional incisions starting from the wound margins along the relaxed skin tension lines.

ii.Subtarsal lower-eyelid approach was used to access the infraorbital rim.

iii.Marking of the subtarsal incision was placed in a natural subtarsal lid crease, about 5 to $7 \mathrm{~mm}$ below the ciliary margin and extended laterally into (or parallel to) one of the resting skin tension lines located along the lateral aspect of the orbit. Following the initial incision through the skin and orbicularis oculi, a preseptal dissection was carried to the level of the orbital rim.

iv.Dissection of the orbit floor up to the posterior edge, medial and lateral boundaries of the fracture releasing the contents that may be herniated into the maxillary sinus including inferior rectus muscle.

v.Lateral eyebrow approach provided simple and rapid access to the superolateral orbital rim.

vi.An approximately $2 \mathrm{~cm}$ long horizontal incision was marked within the bounds of the lateral eyebrow parallel to the hair follicles.

vii.The incision went through the skin first and then through the subcutaneous fat and muscular tissue layers.

viii.The orbicularis oculi muscle was undermined at a level below the retro-orbicularis oculi fat to expose the supraperiosteal plane. The wound edges become freely moveable by the supraperiosteal dissection and are retracted over the frontozygomatic suture or the fracture area 
ix.The periosteum was split sharply along the middle of the superolateral orbital rim with a scalpel. The underlying bony structures are freed using sharp periosteal elevators.

$\mathrm{x}$.After dissection and reduction, the mesh was inserted as follow:

\section{Group 1 (study group)}

The computer assessed titanium mesh was used was adapted without manipulation and fixed with1.5 titanium screws (Fig. 4)

\section{Group 2 (control group)}

Conventional titanium mesh was used. The defect was measured and the mesh was tailored and contoured according to the size of the defect, then the mesh was inserted and fixed by1.5 titanium screws.

xi.Closure of the surgical wound was performed in two layers the muscle was closed using 4/0 polyglactin 910 sutures then insertion of rubber drain was done, and the skin was closed using 6/0 polypropylene sutures.

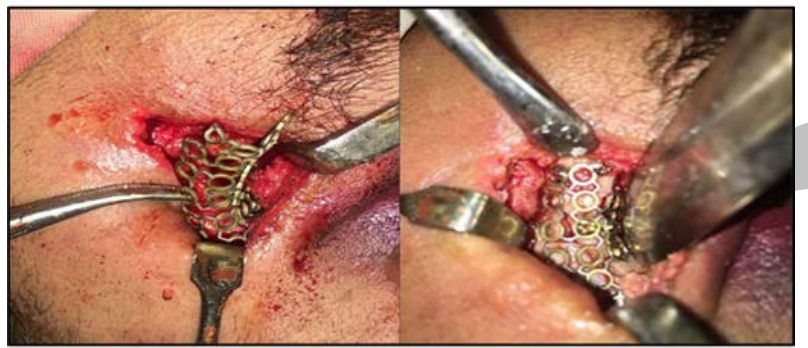

Figure (4): Insertion and fixation of the computer assisted titanium individual mesh

\section{III.Postoperative phase}

\section{A. Post-operative instructions and medications}

- Application of ice / cold packs for 15 minutes of every hour, or at least 4 times a day while patients awake, for the first 3 days.

- All patients were instructed to follow hygienic measures and never blow out for at least 3 weeks.

- All patients continued the same regimen of antibiotics and analgesics for one week postoperatively to control infection and pain. Clindamycin 600mg (Dalacin c phosphate, Upjhon) every 12 hours) and Ibuprofen 400mg every 8 hours (Brufen 400, Abbott).

- Polymyxin/tetracycline eye ointment (Teramycin100IU/g, Polymyxin and 0.5\% oxytetracycline. Pfeizer, Egypt.) 4 times a day until seen in clinic for the post-operative visit.

- Drain was removed after 48 hours.

- Patients were discharged 72 hours after surgery when they are completely free from any immediate postoperative complications including bleeding or retrobulbar hemorrhage.

- Sutures were removed after 7 days.

\section{IV.Follow- up phase}

\section{Clinical evaluation}

- All patients were examined on the 1 st week postoperatively to evaluate the degree of edema, presence of infection, degree of pain, stability of fractured segment, presence of abnormal sensation, and any other clinical findings.

- Pin prick test and electrical pulp testing were performed one month and 6 months postoperatively to evaluate the recovery of the infra-orbital nerve.

\section{Radiographic evaluation}

All patients were radiographically examined on the 1st week, and 6 months postoperatively using computerized tomography (CT) to evaluate healing at fracture line.

\section{Statistical analysis}

- Appropriate statistical analysis was used to evaluate the outcomes of the two groups.

- Means and standard deviations was calculated to describe data collected from the two groups.

- T-test was used to evaluate the two groups to reject or not reject the null hypothesis. The level of significance will be set at $\mathrm{P}<0.05$.

\section{RESULTS}

This study was conducted on twenty patients, (study group) 10 patients underwent orbital reconstruction using the computer-assisted fabricated individual titanium mesh and (control group) 10 patient underwent orbital reconstruction using conventional titanium mesh.

\section{Distribution of age and sex}

The mean age of the study and control group was $34.70 \pm 8.53$ years and $33.50 \pm 9.83$ years respectively, the median was 31 and 30years. In both groups, females represented $20 \%$ of the groups. No statistically significant difference was observed between both groups regarding age and sex $(\mathrm{P}=0.774$ and 1.000).

\section{Fractures site}

In both groups, three patients suffering from isolated lateral orbital wall fracture, two patients with zygomatic complex fracture, two patients with isolated supra orbital wall fracture, one patient with supra orbital rim and roof fracture and two patients with isolated orbital floor fracture.

So the lateral orbital wall fracture represented $30 \%$ of the groups, the lateral orbital wall and orbital floor fracture represented $20 \%$ of the groups, the supra orbital rim fracture represented $20 \%$ of the groups, the supra orbital rim and orbital roof fracture represented $10 \%$ of the groups and orbital floor fracture represented $20 \%$ of the groups.

\section{Neurological evaluation}

\section{Pin prick test}

Preoperatively the affection of infra orbital nerve represented $70 \%$ in both groups. After one and six month the affection was $20 \%$ and $0 \%$ respectively in the study group, $30 \%$ and $20 \%$ respectively in the control group. The difference between the two groups was statistically significant $(\mathrm{P}=0.020$ and 0.005 respectively $)$.

\section{Electrical pulp testing}

Preoperatively the affection of infra orbital nerve represented $30 \%$ in both groups. After one and six month the affection was $20 \%$ and $0 \%$ respectively in the study group, $30 \%$ and $20 \%$ respectively in the control group. The difference between the two groups was statistically significant $(\mathrm{P}=0.020$ and 0.005 respectively.

\section{DISCUSSION}

Fractures of the orbit are among the most common fractures of the midface. As such, there exists a great deal of literature evaluating reconstruction of this region. There is still much debate about many aspects of the treatment of these injuries given the multitude of available materials and techniques for reconstruction (9).

In this study mirroring-reconstruction of the orbital walls using computer-assisted individually fabricated 
titanium mesh was a more definitive solution to restoring the orbit anatomy to the pre-injury level and allowed precise adaptation of the titanium mesh with respect to important landmarks (e.g., posterior ledge, ethmoidal bulge), which were often difficult to determine intraoperatively.

Traditionally, intraoperative trimming of the mesh can lead to multiple "try ins" that can traumatize delicate soft tissue with poor adaptation and positioning, often necessitating revision procedures (10).

In our study group cases computer -assisted titanium orbital mesh was placed almost immediately with minimal or no further manipulation or adaptation without risk of soft tissue trauma. This was because virtual reduction created on rapid prototyping (RP) orbital model reduced the trial and error that was normally required for open reduction. The bony injuries were analyzed in all planes and virtual reduction of the fractures was carried out in all study group cases top down, outside in, so the operating time was reduced and allowed precise positioning. Planning of the surgery started with a meeting with the biomedical engineer of the modeling company, during the meeting, the fractured segments were virtually reduced to optimize bony alignment, volumetric analysis of the reduced segments was easily computed by the virtual software which aided in establishing reduction.

Regarding the parenthesis of the infraorbital nerve, we reported that, the superiority of reduction and the use of computer- assisted titanium mesh preventing sensory deficit. By two different procedures we evaluated the function of the infra orbital nerve which included pin prick test and electrical pulp testing we found that, there was significant difference between the two groups $(\mathrm{P}=0.020$ and 0.005 respectively). This in agreement with the study of Ozer et al 2016 (11) who reported that the recovery of the infra orbital nerve function need precise reduction he was evaluated with two different procedures which included electrical detection threshold and pin prick method. At 1 month time, post operatively $50 \%$ patients having electrical hyperesthesia when test with electrical detection threshold. It was seen that at all the locations the mean electrical threshold was higher at affected site as compared to control site representing hypoesthesia. The differences between two sites were found to be significant statistically $(p=0.05)$. Although there is no international consensus on the ideal material to be used for orbital reconstruction (11). However, in our study the success of orbital fracture repair depends not only on the use of material that restores the premorbid orbital volume and shape, but also the capacity to visualize the geometry of the bony defect spatially and to place the titanium mesh to fit the original contour with no evidence of inflammatory and fibrogenic response to the surrounding tissue. This in agreement with Kim, et al 2017 (12) and Ellis et al 2004 (13). Whose reported that, titanium mesh has been cited as being an excellent alloplastic material for use within the orbit as it has a low rate of extrusion and infection which has been attributed to the tendency of titanium to cause an inflammatory and fibrogenic response to the surrounding tissue. Titanium has been shown to cause increased levels of fibroblast activity especially around grooves and ridges Mwenifumbo, et al 2007 (14), as well as increased levels of transforming growth factor- $\beta$ and platelet derived growth factor.

Therefore, our study reported that, implementation of individually designed titanium mesh completely meets the requirements of orbital reconstruction. Fabrication of titanium mesh using three-dimensional printed models, with previous preparation based on computer aided design (CAD) and rapid prototyping (RP) technologies, allowed better positioning and adhesion of the mesh within area of defect, almost which a prerequisite for prevention of paresthesia of the infraorbital nerve, Although patientspecific titanium mesh seem to offer the best reconstructive contour, their fabrication was expensive while the decreased complications and generalized improved outcomes seen signify cost savings that may offset the technological costs.

\section{CONCLUSION}

From this study we concluded that, the use of patient specific 3D-sterioleithography rapid prototyping orbital models and prefabricated individual titanium mesh was an accurate option in reconstruction of traumatic orbital wall fractures. It restored the anatomy of the orbit, eliminating time spent and successful clinical outcome had been obtained.

\section{CONFLICT OF INTEREST}

The authors declare that they have no conflicts of interest.

\section{REFERENCES}

1-Bevans SE, Moe KS. Advances in the reconstruction of orbital fracture. Facial Plast Surg Clin N Am. 2017;25:513-35.

2-Talwar RM, Chemaly D. Information and computer technology in oral and maxillofacial surgery. Oral Maxillofac Surg Clin North Am. 2008;20:79-89.

3- Edwards SP. Computer-assisted craniomaxillofacial surgery. Oral Maxillofac Surg Clin North Am. 2010;22:117-34.

4-Lim CG, Campbell DI, Clucas DM. Rapid prototyping technology in orbital floor reconstruction: application in three patients. Craniomaxillofac Trauma Reconstr. 2014;7:143-6.

5-Cohen A, Laviv A, Berman P, Nashef R, Abu-Tair J. Mandibular reconstruction using stereolithographic 3dimensional printing modeling technology. Oral Surg Oral Med Oral Pathol Oral Radiol Endod. 2009;108:661-6.

6-Yu AW, Khan M. On-demand three-dimensional printing of surgical supplies in conflict zones. J Trauma Acute Care Surg. 2015;78:201-3.

7- Prachur Kumar, Godhi S, Lall AB, Ram CS. Evaluation of neurosensory changes in the infraorbital nerve following zygomatic fractures. J Maxillofac Oral Surg. 2012;11:394-9.

8-Chen E, Abbott PV. Abbott dental pulp testing: A review. Int J Dent. 2009;2009:1-12.

9- Gordon CR, Susarla SM, Yaremchuk MJ. Quantitative assessment of medial orbit fracture repair using computerdesigned anatomical plates. Plast Reconstr Surg. 2012;130:698-705.

10-Stoetzer M, Rana M, von See C, Eckardt AM, Gellrich NC. Reconstruction of defects of maxillary sinus wall after removal of a huge odontogenic lesion using prebended 3D titanium-mesh and CAD/CAM technique. Head Face Med. 2011;7:1-3.

11-Ozer MA, Govsa F, Kazak Z, Erdogmus S, Celik S. Redesign and treatment planning orbital floor reconstruction using computer analysis anatomical landmarks. Eur Arch Otorhinolaryngol. 2016;273:2185-91. 
12-Kim YC, Jeong WS, Park T-k, Choi JW, Koh KS, Oh TS. The accuracy of patient specific implant prebented with 3Dprinted rapid prototype model for orbital wall reconstruction. J Craniomaxillofac Surg. 2017;45:928-36.

13-Ellis E 3rd, Messo E. Use of nonresorbable alloplastic implants for internal orbital reconstruction. J Oral Maxillofac Surg. 2004;62:873-81.

14-Mwenifumbo S, Li M, Chen J, Beye A, Soboyejo W. Cell/surface interactions on laser micro-textured titaniumcoated silicon surfaces. J Mater Sci Mater Med. 2007;18:9-23.

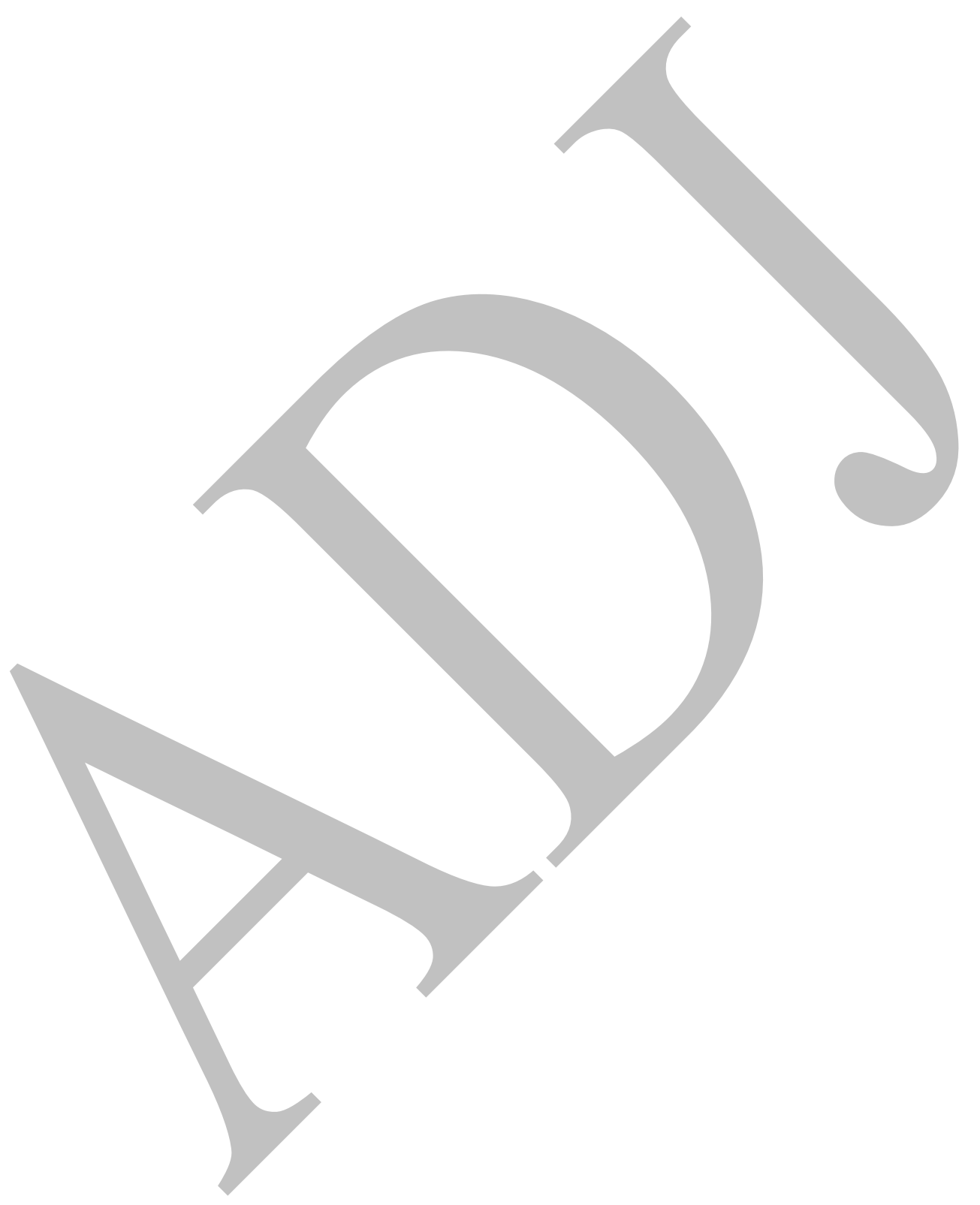

